(C) IEEE

Madina, Carlos, Ines Gomez-Arriola, Maider Santos-Mugica, Joseba Jimeno, Kris Kessels, Dimitris Trakas, Jose Pablo Chaves, and Yvonne Ruwaida. "Flexibility Markets to Procure System Services. CoordiNet Project." 2020 17th International Conference on the European Energy Market (EEM) (September 2020). doi:10.1109/eem49802.2020.9221890.

https://doi.org/10.1109/eem49802.2020.9221890 


\title{
Flexibility markets to procure system services. CoordiNet project
}

\author{
Carlos Madina, Inés Gómez-Arriola, Maider Santos-Mugica, Joseba Jimeno \\ TECNALIA, Basque Research and Technology Alliance (BRTA) \\ Spain \\ Kris Kessels \\ VITO/EnergyVille \\ Belgium \\ José Pablo Chaves \\ Universidad Pontificia Comillas \\ Spain \\ Dimitris Trakas \\ National Technical University of Athens \\ Greece \\ Yvonne Ruwaida \\ Vattenfall Distribution AB \\ Sweden
}

\begin{abstract}
The efficiency and reliability of electricity systems depend, among other aspects, on an efficient collaboration between the different market participants, which require updating the roles of all agents involved. The CoordiNet project, co-funded by the EU, intends to demonstrate how TSOs and DSOs can act in a coordinated manner, to purchase and activate system services, promote the cooperation of all actors and eliminate barriers for the active participation of DERs in the market. The results of the project will help to design scalable tools and methodologies for system operators and third parties to safely connect, manage and coordinate flexibility providers. This paper describes the developments in CoordiNet to ensure the interoperability of the different markets and platforms developed by TSOs and DSOs across Europe.
\end{abstract}

Index Terms--Flexibility, platform, demonstrator, TSO-DSO cooperation

\section{INTRODUCTION}

The Directive 2019/944 on common rules for the internal market for electricity [1] promotes that network operators procure system services, including balancing and congestion management, from assets connected to both transmission and distribution networks, based on a cooperation among them. As a result, many research projects are investigating how to improve the cooperation between TSOs and DSOs for the procurement of system services, e.g. SmartNet (http://smartnetproject.eu/), and even a specific topic (LC-SC3-ES-5) was included in the H2020 programme [2]. The selected projects under this topic were Interrface (http://www.interrface.eu/) and CoordiNet (https://coordinet-project.eu). The purpose of the CoordiNet project is to demonstrate how Distribution System Operators (DSOs) and Transmission System Operators (TSOs) shall act in a coordinated manner to procure and activate system services in the most reliable and efficient way through the implementation of three large-scale demonstrations. By achieving this, CoordiNet intends to contribute to the development of a smart, secure and more resilient energy system. A detailed analysis on the definition of flexibility in the grid at every voltage level ranging from the TSO and DSO domain to consumer participation will be performed. The project aims to demonstrate how DSOs and TSOs, acting in a coordinated manner, can provide favourable cooperation conditions to all actors, removing barriers to the participation of all resources, including small Distributed Energy Resources (DERs). In addition, CoordiNet will also develop new mechanisms, more suitable for real-time operation, in order to define requirements for the development of interoperable European platforms in the future. The results will allow the definition of scalable tools and methodologies for system operators and third parties to safely connect, manage and coordinate with flexibility providers.

\section{DEMONSTRATION PROJECTS}

The CoordiNet project gathers the efforts of 23 companies from ten European countries to test the mechanisms to be developed in three large-scale demonstration projects across ten different locations in Greece (Kefalonia and Mesogia), Spain (Albacete, Cádiz, Málaga and Murcia-Alicante), and Sweden (VästerNorrland-Jämtland, Uppland, Gotland and Skåne). They will apply the coordination schemes and will test the products and system services defined within the project. Lessons learned from these demonstration projects will provide insights on how to realize interoperable coordination platforms across Europe.

\section{A. Greek demonstrator}

The Greek demonstrator aims at: i) preparing consumers and renewable energy sources to acquire a more active role in the management and operation of the power system at national 
and regional level, ii) creating new products and services to provide a reduced cost of energy and improved quality of supply to consumers, and iii) utilize existing operational and demonstration systems with new ones developed in the project.

The demonstrator will be deployed in two different regions, as shown in Figure 1.

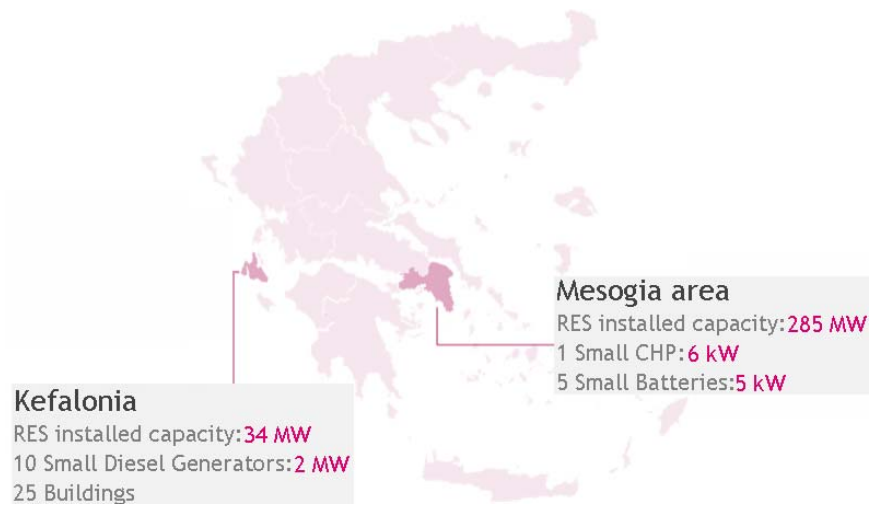

Figure 1: Greek demonstrator

\section{B. Spanish demonstrator}

The demonstrator aims to proof the technical and economic viability of a system that allows flexibility providers, regardless of their size and voltage level of their connection point, to provide system services to both DSOs (to solve congestions, voltage and islanding operation problems) and the TSO (to solve congestions, voltage and balancing problems). Figure 2 shows the locations of the demonstrator.

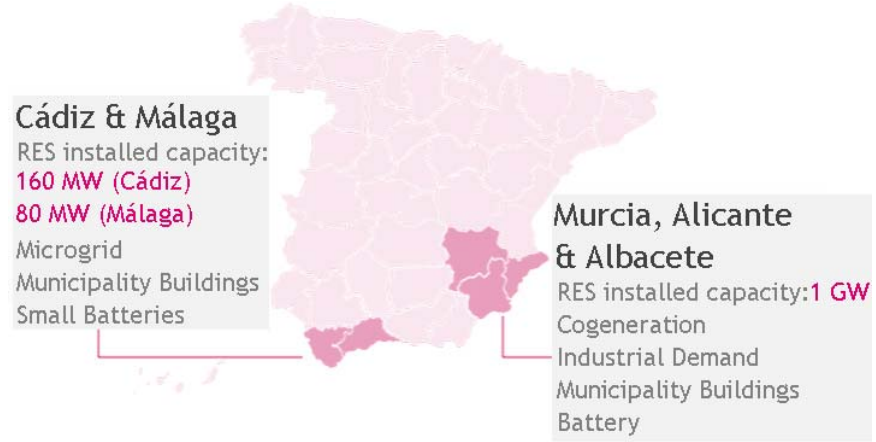

Figure 2: Spanish demonstrator

\section{Swedish demonstrator}

The Swedish demonstrator is expected to promote economic growth and electrification to achieve a fossil-free society, through the use of grid capacity in a more flexible way over time. The established flexibility market allows the flexibility providers to offer capacity to the DSOs. The established flexibility products will be merged into the DSO's operation and grid planning procedures, as well as customers' practices. The new TSO-DSO coordination will be improved by real-time data gathering and forecasting tools for grid and customer behaviours. The demonstrator will contribute to the national and local climate and renewables' goals, as well as to the economic utilization of assets. Moreover, different DERs, such as storage, wind and small-scale customers will participate in the flexibility market, via aggregation. A digital peer-to-peer (P2P) market will be in place to enable trading, planning and economical transactions. Figure 3 shows the location in the four different regions.

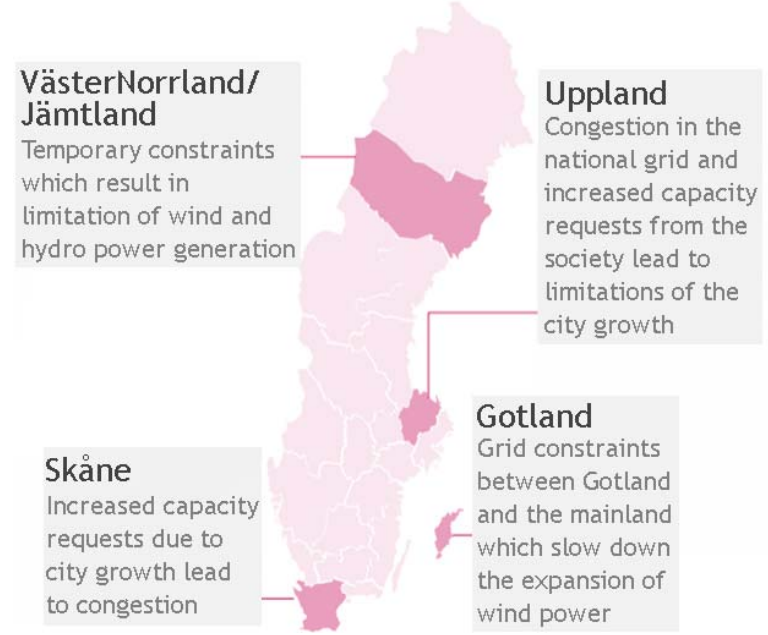

Figure 3: Swedish demonstrator

\section{SYSTEM SERVICES AND COORDINATION SCHEMES}

System services enable system operators to operate the transmission and distribution grids in a secure manner. In CoordiNet, these services are defined as services provided to DSOs and TSOs to keep the operation of the grid within acceptable limits for security of supply and which are delivered mainly by third parties (adapted from [3]). CoordiNet considers balancing, congestion management, voltage control and controlled islanding.

In order to enable a market-based allocation of these system services and, thus, allow market parties to effectively bid into these markets, products for such services need to be defined. First, standard products were defined for the three demo regions (Greece, Spain, Sweden), which are harmonized products for the exchange of system service(s) with common characteristics across Europe (i.e. shared by all TSOs or by all DSOs or by all TSOs and DSOs). Then, local circumstances were considered to adapt some system services (e.g. congestion management) and specific products were defined.

After defining the services and products, different alternatives for TSO and DSO coordination need to be specified. This coordination is required to ensure an optimal use of the inherent flexibility of DERs and, thus, the market design must allow a close collaboration between both system operators. Since there is no single solution that fulfils the needs of all power systems (due to local circumstances, market maturity or regulatory conditions) there are many possible TSO-DSO coordination schemes. Specifically, in CoordiNet, the coordination schemes considered are [4]:

1) Local market model: The DSO buys flexibility to solve a local need in one market and no interaction with the central flexibility needs is considered.

2) Central market model: The TSO acquires flexibility solely to solve a central need in a single market. This market is 
operated by the TSO, regardless of whether the resources are connected at transmission or distribution level.

3) Common market model: Both local and central needs coming from DSO and TSO are considered in a single market and, thus, both system operators can use assets that are connected to the distribution grid. The goal of the market is to optimize the outcome of the system as a whole.

4) Multi-level market model: In this case, both local and central needs are solved, but each system operator manages its own market, rather than through a single market for both. In addition to the DSO, the TSO has access to DERs to address their need for flexibility. Bids from DER that are not selected and not procured at the local market, can participate in the central market where the resources connected to the transmission grid participate.

5) Fragmented market model: The market is split as in the Multi-level Market Model, but the TSO has no access to DERs. Therefore, resources connected to the distribution grid can only offer their flexibility in the market managed by the DSO.

6) Integrated market model: This coordination scheme also solves local and central needs, but, in addition to the TSO and the DSO, other market agents are allowed to participate in order to acquire flexibility, both from resources coonected at transmission and distribution level, in a single market.

7) Distributed market model: In this market model, either a local need or both local and central needs are solved through a P2P trade among DERs, which are the only sellers and buyers in the market. DSOs and TSOs set the rules and mechanisms that align peers' self-interest objectives with global objectives.

\section{BusInEsS USE CASES}

Once the products, services and coordination schemes are defined, Business Use Cases (BUCs) for each demonstrator can be defined. In CoordiNet, for each demonstrator, a BUC has been defined for each service and coordination scheme combination. Each service will be tested with different timeframes, in different market models and by using different product types. As a result, the BUCs cover timeframes from long-term planning up to near real-time operations and the system services are offered via central, local, common, multilevel, fragmented and distributed market mechanisms as shown in Figure 4 (updated from [5]). Note that the integrated market approach is out of scope of the demonstrators. As it can be seen in Figure 4, at least one of the services will be tested under two different market models in each demonstrator:

- In Greece, the congestion management and voltage control services will be tested using two different coordination schemes: the multi-level and the fragmented market models.

- In Spain, the congestion management service will be tested for the local and the common market models.

- In Sweden, the focus will be on using a flexibility service for congestion management, allowing flexibility providers to use the same asset also to provide system services, in the multi-level and distributed market models.

Thereby, the advantages and disadvantages of the different setups can be identified. For example, local markets may suffer from lower level of competition than common markets, but they may also facilitate the participation of small-scale flexibility providers, allowing a higher exploitation of the flexibility potential and, thus, increasing market liquidity in locations where there are enough small flexibility providers. This is especially important for congestion management, but it may also be relevant for balancing in semi-isolated areas with limited interconnection capacity (e.g. Gotland).

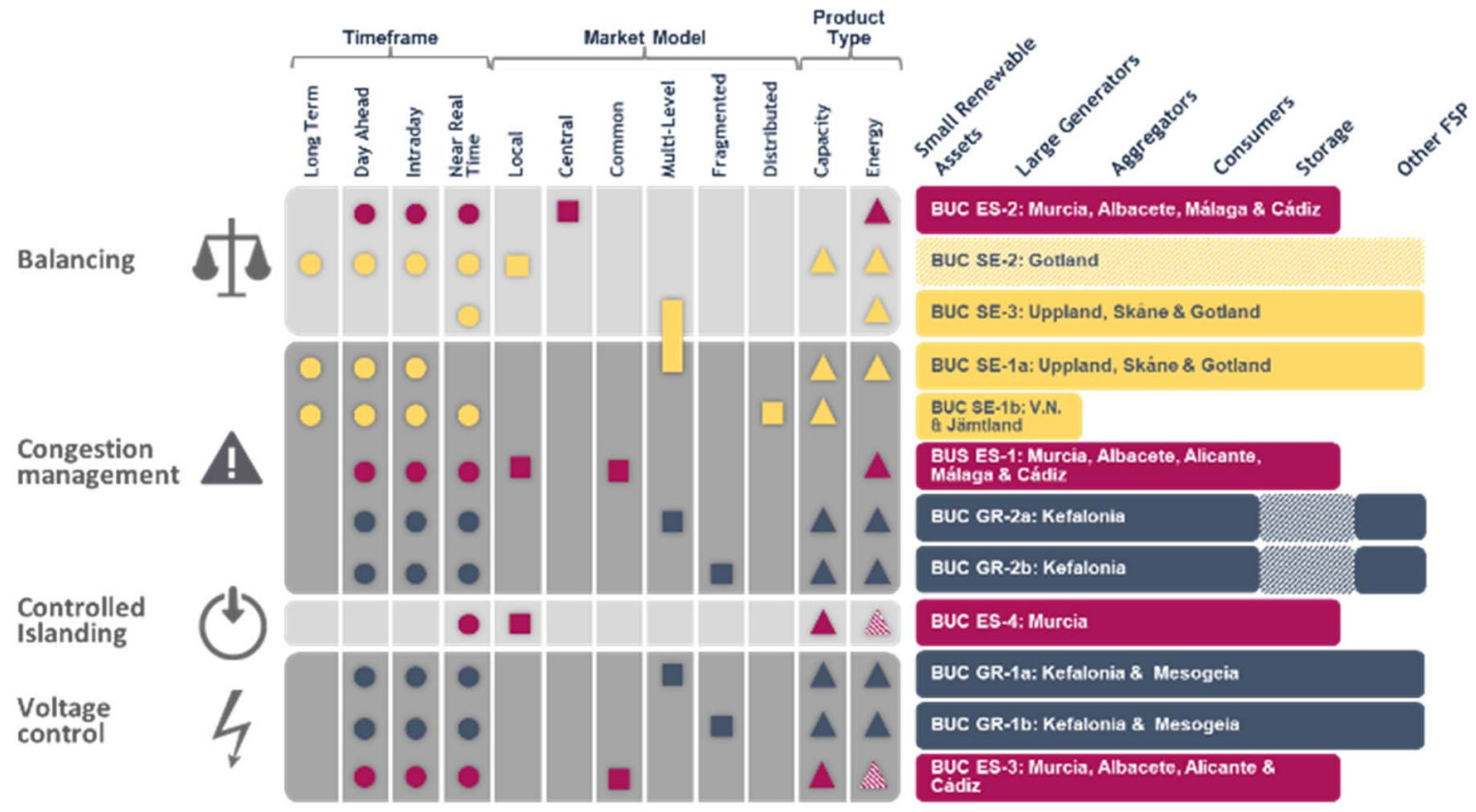

Figure 4: CoordiNet BUCs 


\section{PlatForms For Procuring System SERVICES}

European countries have advanced in the harmonization of the electricity markets, achieving important milestones such as the implementation of market coupling for the forward, dayahead and the continuous intraday markets. This process is also being applied to balancing markets, as the Electricity Balancing Guideline (EBGL) [6] requires. As a result of the implementation of the EBGL, different TSO-TSO platforms are being developed to create a harmonised level-playing field for all market participants across Europe and to improve the procurement of different balancing products by establishing cross-border procurement procedures. Yet, the strong increase of DER deployment has led to a different operation of current electricity systems. Thus, distribution networks will demand a closer collaboration between TSOs and DSOs and, hence, the creation of TSO-DSO platforms is an emerging topic in Europe.

In this context, the CoordiNet project is also defining the characteristics that future interoperable platforms for the procurement of system services should have. The CoordiNet platform is an interface that is intended to manage different interactions between the TSO, DSOs and the Flexibility Service Providers (FSPs) to coordinate the different functions necessary to perform the BUCs.

The deployment of the CoordiNet platform implies:

- Definition of characteristics, functionalities and potential algorithms (market clearing, generation and demand forecasting, aggregation, etc.), in order to ensure the interoperability of markets and platforms developed by TSOs and DSOs across Europe.

- Coordination among market platforms and the demonstrators in order to develop various solutions which enable them to test their specific innovative standardized products and to define how the platforms interact with existing market designs.

- Specification and development of common interfaces between the various modules of the platform.

- Identification of potential gaps and needs of alignment among the demonstrators to start the standardization process of future system services and market platform solutions (including innovative technologies).

The CoordiNet platform implies the development of several modules, as well as the interfaces linking these modules: markets for system services, tools for monitoring and operating systems and aggregation/disaggregation modules.

\section{A. Platform in the Spanish Demonstrator}

In the case of the Spanish demonstrator [7], the CoordiNet platform will be divided into a common platform, managed by the Spanish TSO (Red Eléctrica de España, REE) and a local platform, managed by e-distribución and i-DE, the DSOs participating in the Spanish demonstrator.

REE will develop and host the common platform within the CoordiNet Platform concept. This common platform includes some existing systems, which will be adapted, and a brand new interface that will allow for a more efficient communication between REE and the DSOs. The platforms already available, their expected modifications and new developments are:

1) Control Centre of Renewable Energies (CECRE): The CECRE, is the control centre devoted to renewable generation. This control leads to improved security and effectiveness in system operation allowing higher energy productions for the same installed capacity and a more efficient real-time operation of the plants. The CECRE will be the gateway between the generation units and the TSO, both for reception of monitoring data and for sending set-points, but no major modification is foreseen within CoordiNet.

2) GEMAS+: It accesses the real-time information received in CECRE and uses it to assess whether the present generation scenario is admissible for the system due to several criteria. When it is necessary to return to a N-1 secure state, GEMAS+ calculates the amount of power reduction, minimizing the overall cost for the system. GEMAS+ generates a file with the set-points (and their motivations) for each generator and this information is sent to the System Operator's Information System and the Renewable Energy Control Centre (RESCC), centres for the real-time information collection from the RES plants linking these installations to the CECRE and acting as the only real-time interlocutor with the TSO. Several adpatations will be performed, so that the TSO can run the congestion management market considering constraints highlighted by the DSO in its network through the CoordiNet Platform Interface. This application will use as much as possible the market structure and algorithms already used by the TSO, although new developments will be required for the voltage control service, since this service is not provided under a market framework yet in Spain.

3) E-SIOS: The System Operator Information System (E-SIOS), is specially designed to run all the necessary processes to ensure an economic and reliable exploitation of the Spanish Power System in real-time. The bids for the balancing markets are managed through E-SIOS. The current system will be slightly modified to accommodate the new markets called by the DSO through the CoordiNet Platform. ESIOS will also be the interface between the common platform and the market agents (FSPs). As for GEMAS+, several developments are required for the voltage control use case.

4) CoordiNet Platform Interface: It consists of a new platform that allows DSOs to call markets for different needs, based on the structural information already in place in the GEMAS+ system. Therefore, the DSOs will be able to call for congestion management markets and set the limits to the participation in balancing markets to resources connected in their networks when congestions occur, whenever needed and for different locations. A common market will be run in a coordinated manner with the TSO. It is expected that the DSO will run the local market just after the common congestion management market.

Figure 5 summarizes the different systems considered for the common platform and their interaction regarding the congestion management processes. 


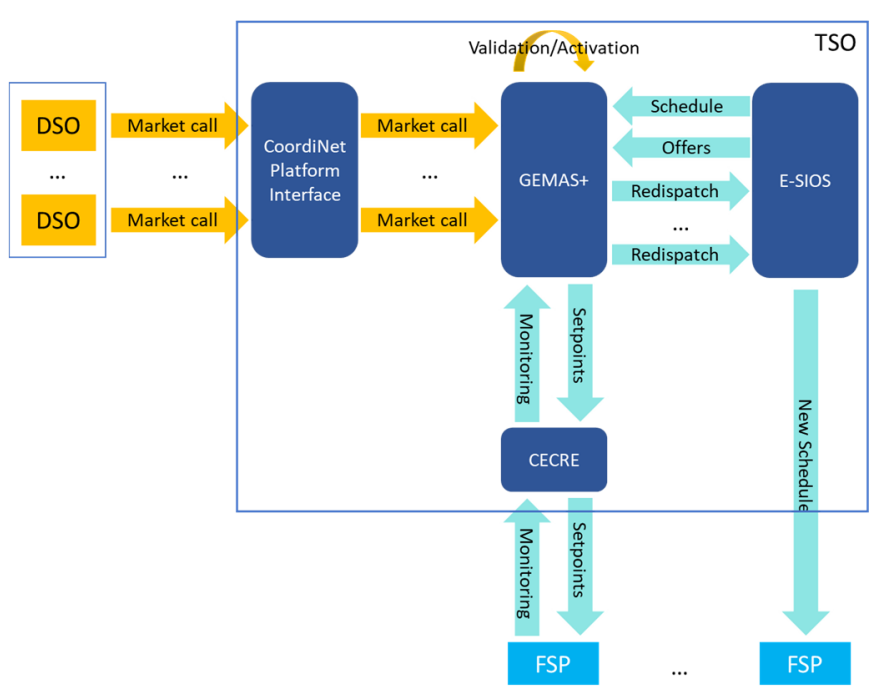

Figure 5: CoordiNet common platform architecture [7]

Regarding DSOs, the current system monitoring depends on the level of observability and controllability of the networks, which depends on the voltage level. Likewise, the DSOs have grid architecture criteria to define the necessity of monitoring and control points, both for generators and demand.

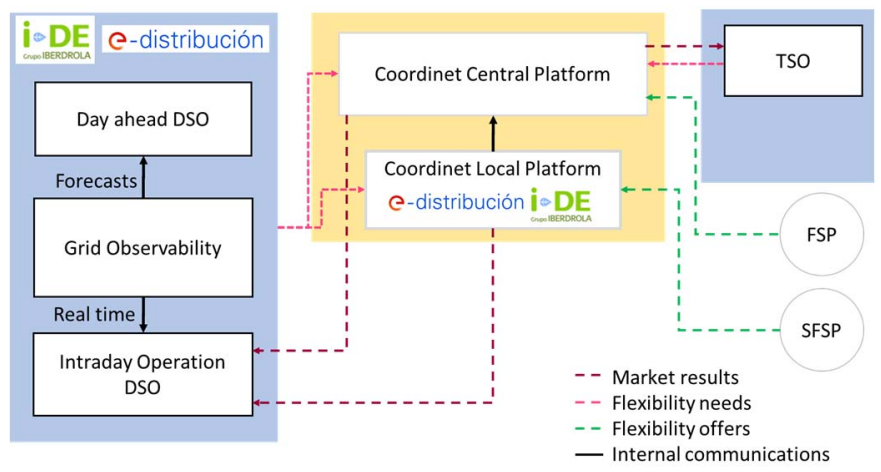

Figure 6: DSO Platform Structure [7]

A DSO platform is being built, composed of several modules (see Figure 6), with the following functionalities:

1) Day-ahead DSO: This module is in charge of sending the needs from distribution networks to different platforms. The platform also allows registering manual requirements or inputs from other DSOs' platforms.

2) CoordiNet Local Platform: This local platform receives bids from small FSPs (supply) and DSOs (demand). Then, the market clearing algorithm is executed, and the matched offers and corresponding activations are sent to the providers, to the common platform and to the intraday operation submodule.

3) Intraday Operation DSO: The intraday operation module is in charge of checking the results computed in the market clearing, where the flexibility services can be traded.

4) Grid Observability: This module is related to the operation of the grid. It includes the forecasting, metering and checking of activation functionalities, which are essential for the correct operation of the market module.
5) Communications: This module is responsible for establishing all communications between the local platform, the common platform, the DSO platform, and the small FSPs.

\section{B. Platform in the Swedish Demonstrator}

In the new, day-ahead congestion management market already demonstrated in Sweden, DSOs run the flexibility market (first, local DSOs and, then, regional DSOs) before the day-ahead market. The decisions on activation for congestion management purposes are taken by the operators in the control centre. based on continuous analysis of the system state, including parameters such as frequency, real-time flows, production and consumption plans. The activation is done by mail from the platform, but electronic activation (through Application Programming Interfaces, APIs) is expected to be implemented during 2020. No specific communication protocol is adopted yet, but the Energy Communication Platform is foreseen to replace the manual activation by phone. In winter 2020 , this market will be combined with the mFRR market, allowing FSPs to participate in the provision of both services through the platform [8].

A modular and adaptable approach (Figure 7, more details in [9]) has been developed, using the Azure cloud environment as a basis, to align software development of the platform with the BUCs and their requirements. The technology stack consists of modern tools selected for their versatility and relative speed. The platform interfaces with several different APIs to collect and send data, such as internally used power meters, Grafana telemetry data for monitoring status of the platform, weather observational data, energy metering of substations and production plants from SCADA, external data from FSPs and TSO/DSOs sent to the web API of the platform backend, etc.

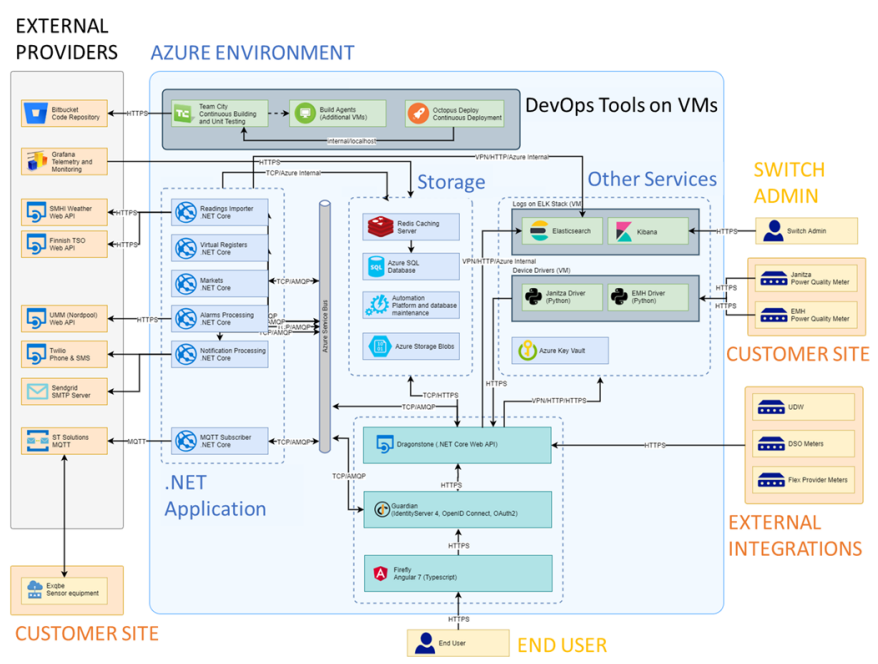

Figure 7: High-level architecture platform [9]

E.ON Distribution and Vattenfall Distribution, use slightly different approaches for collecting the measurements from FSPs in the demonstration activities. At E.ON, the flexibility market platform receives asset power data from external meters when available, and the settlement is based on both external and internal meter data. Vattenfall uses IoT (Internet of Things) boxes to interface with existing meters and these data are sent to the flexibility market platform as well as to internal servers. 


\section{Platform in the Greek Demonstrator}

Currently, there is no platform for congestion management nor voltage control in Greece (services to be tested in the Greek demonstrator) at transmission nor distribution levels. Besides, there is no communication platform between IPTO (the TSO) and HEDNO (the DSO). Therefore, the CoordiNet platform under development is constituted by TSO-DSO collaboration and market platforms. One major contribution will be the provision of alternative solutions to system expansion, in order to tackle the problems arising by the existing and forthcoming high RES penetration in the considered areas [8].

The Greek demonstrator will deploy the multi-level and fragmented market models for the implementation of the corresponding functions necessary to perform the BUCs. To that end, the required market platforms will be developed from scratch in order to: a) handle and match the bids from the producers or FSPs, with the DSO and TSO flexibility request, b) clear the different markets (day-ahead, intraday, near realtime) and c) extract the market results for all market timehorizons [10]. In addition, the TSO/DSO communication platform will be deployed to allow the system integration and information exchange between the TSO and DSO operation and information technology systems, by using an Enterprise Service Bus (ESB) approach. Figure 8 (adapted from [11]) shows the platforms and tools to be deployed in the Greek demonstrator.

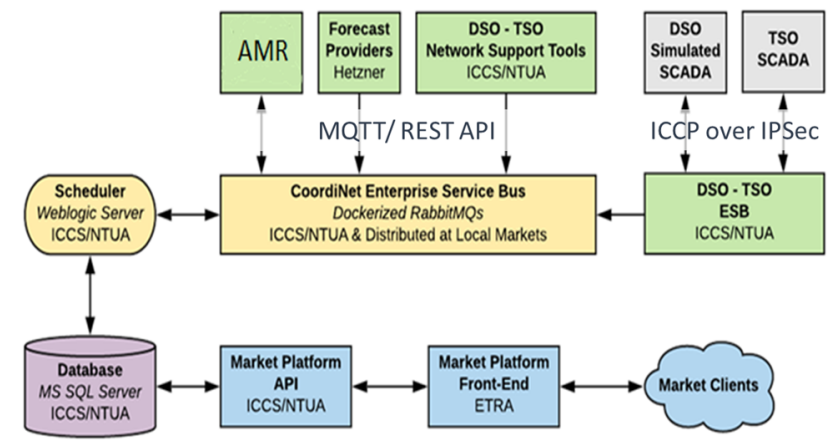

Figure 8: TSO/DSO platforms and tools in the Greek demonstrator

\section{CONCLUSIONS}

The CoordiNet project aims to demonstrate how DSOs and TSOs shall act in a coordinated manner to procure system services in the most reliable and efficient way. With that purpose, the demonstrators in Greece, Sweden and Spain develop innovative approaches. In Greece, the main contribution will be the provision of alternative solutions to system expansion, in order to tackle the problems arising by the existing and forthcoming high RES penetration in the areas in which the demonstrator is arranged. In Spain, three of the main challenges are that the demo considers demand side resources, whose participation in the market is currently limited, the improvement of the process to solve congestions at distribution level by organising a common congestion management market, and the increase of the network observability by the DSOs, specially at low-voltage levels. In Sweden, the use of local flexibility solutions aims to alleviate network congestions and avoid power shortages. On this basis, the deployment of novel local markets is expected to complement existing central markets for ancillary services resulting in an equilibrium taking also regional constraints into account as a pricing factor.

The flexibility service for day-ahead congestion management has been successfully demonstrated in Sweden during the winter 2019/2020 and lessons have been extracted [12] for the second run, which will start in late 2020. Likewise, final arrangements are being made to start the first demo runs in Greece and in Spain in the winter 2020 season, so no result is available yet. In parallel, the characteristics of the main systems that need to exchange information through the CoordiNet platform (the TSO and DSO systems for grid monitoring and operation, the systems for market clearing and the tools and systems used for aggregation and disaggregation functions) are being collected and analysed, in order to identify the data exchange needs, so that the interfaces of the CoordiNet platform can be standardized.

\section{ACKNOWLEDGMENT}

This project has received funding from the European Union's Horizon 2020 research and innovation programme under grant agreement No 824414 .

\section{REFERENCES}

[1] "Directive (EU) 2019/944 of the European Parliament and of the Council of 5 June 2019 on common rules for the internal market for electricity and amending Directive 2012/27/EU”. Official Journal of the European Union 158, 14.6.2019, p. 125-199.

[2] "Horizon 2020 Work Programme 2018-2020, 10. Secure, clean and efficient energy" European Commission Decision C(2020)1862 of 25 March 2020.

[3] CEDEC, EDSO, ENTSO-E, Eurelectric, GEODE. "TSO-DSO Report An integrated approach to active system management with the focus on TSO-DSO coordination in congestion management and balancing". 2019.

[4] A. Delnooz, J. Vanschoenwinkel, E. Rivero, C. Madina. CoordiNet deliverable 1.3, "Definition of scenarios and products for the demonstration campaigns". July 2019.

[5] G. Gürses-Tran, G. Lipari, D. Trakas, M. Santos-Múgica, J. P. Chaves, K. Kessels, Y. Ruwaida, M. Uslar. CoordiNet Deliverable 1.5, "Business Use Case definition”. July 2019.

[6] Commission Regulation (EU) 2017/2195 of 23 November 2017 establishing a guideline on electricity balancing. Official Journal of the European Union 312, 28.11.2017, p. 6-53.

[7] J. P. Chaves, T. Gómez, L. Lind, M. A. Sánchez, L. Olmos. CoordiNet Deliverable 3.1, "Report of functionalities and services of the Spanish demo". January 2020.

[8] J. P. Chaves, L. Lind, G. López, J. Matanza, D. Trakas, I. Vlachos, G. Lipari, M. Santos, I. Gómez. CoordiNet Deliverable 2.0, "Coordination and knowledge sharing between demonstration campaigns on market architectures" (Unpublished).

[9] D. Bjarup, C. Isendahl. CoordiNet Deliverable 4.4. "Finalized small scale demo run - functionalities, products and routines". April 2020.

[10] T. Bachoumis, P. Dratsas, C. Kaskouras, M. C. Sousounis, L. Viguer, A. I. Martínez, I. Vlachos, A. Dimeas, D. Trakas, A. Botsis, G. Sideratos. CoordiNet Deliverable 5.1. "Demonstrator. Analysis \& Planning". January 2020.

[11] CoordiNet Deliverable D5.2, "Report on Software Development Activities for the Greek Pilot" (Unpublished).

[12] N. Etherden, Y. Ruwaida, S. Johansson. CoordiNet Deliverable D4.5. "Report on lessons learned, bug fixes and adjustments in products and routines within the Swedish demo". June 2020.

All CoordiNet deliverables are available at https://coordinetproject.eu/publications/deliverables. 